\title{
Treating Surfaces with a Cold Atmospheric Pressure Plasma using the COST-Jet
}

\author{
Judith Golda ${ }^{1}$, Kerstin Sgonina ${ }^{1}$, Julian Held ${ }^{2}$, Jan Benedikt ${ }^{1}$, Volker Schulz-von der Gathen ${ }^{2}$ \\ ${ }^{1}$ Experimental Plasma Physics, Kiel University ${ }^{2}$ Experimental Physics II, Ruhr-University Bochum
}

\section{Corresponding Author}

Judith Golda

golda@physik.uni-kiel.de

\section{Citation}

Golda, J., Sgonina, K., Held, J.,

Benedikt, J., Schulz-von der

Gathen, V. Treating Surfaces with a Cold Atmospheric Pressure Plasma using the CosT-Jet. J. Vis. Exp. (165), e61801, doi:10.3791/61801 (2020).

\section{Date Published}

November 2, 2020

DOI

$10.3791 / 61801$

URL

jove.com/video/61801

\section{Abstract}

In recent years, non-thermal atmospheric pressure plasmas have been used extensively for surface treatments, in particular, due to their potential in biological applications. However, the scientific results often suffer from reproducibility problems due to unreliable plasma conditions as well as complex treatment procedures. To address this issue and provide a stable and reproducible plasma source, the COSTJet reference source was developed.

In this work, we propose a detailed protocol to perform reliable and reproducible surface treatments using the COST reference microplasma jet (COST-Jet). Common issues and pitfalls are discussed, as well as the peculiarities of the COST-Jet compared to other devices and its advantageous remote character. A detailed description of both solid and liquid surface treatment is provided. The described methods are versatile and can be adapted for other types of atmospheric pressure plasma devices.

\section{Introduction}

Cold atmospheric pressure plasmas (CAPs) have attracted increased interest in recent years due to their potential for surface treatment applications. CAPs are characterized by their non-equilibrium properties, enabling complex plasma chemistry with a high density of reactive species while maintaining a low thermal impact on treated samples. Therefore, CAPs are considered in particular for the treatment of biological tissue ${ }^{1,2,3,4}$. Numerous concepts and designs of CAPs are successfully used for wound disinfection and healing, blood coagulation, and cancer treatment, among other biomedical applications. A large proportion of biological tissue contains liquids. Therefore, research is also increasingly focused on investigating the effects of CAPs on liquid surfaces such as cell medium or water ${ }^{5,6,7}$.

However, the scientific results often suffer from reliability and reproducibility problems $8,9,10$. On one hand, the treated biological substrates are subject to natural variations. On the other hand, biological mechanisms were seldom directly attributed to plasma processes (such as electric fields, UV radiation, and long- and short-lived species, etc.). 
Furthermore, these plasma processes in turn depend strongly on the individual plasma source and the exact type of its application.

Additionally, detailed protocols of treatment procedures are rarely available. This makes it difficult to isolate the influence of a particular plasma parameter on the outcome of the treatment, which renders the obtained results nontransferable.

Therefore recently, various attempts have been made to standardize the treatment of surfaces, tissues, and liquids using cold atmospheric pressure plasmas. Here we present only some selected examples.

1. To simplify the direct comparison of different plasma sources, a reference source was developed. Inspired by the low-pressure plasma community, a reproducible and stable discharge design (COST-Jet) was developed in the framework of the COST action MP 1101 that can serve as a reference source for future biomedical research ${ }^{11}$.

2. To enable comparability, reference protocols for individual applications were developed. To standardize the comparison of the antimicrobial properties of cold atmospheric pressure plasmas, for example, Mann et al. defined a reference protocol for microorganism treatment by normalizing the treatment time per area unit ${ }^{12}$.

3. For a more flexible approach, Kogelheide et al. developed a method to investigate plasma-induced chemical modifications on macromolecules ${ }^{13}$. Using tracer compounds such as cysteine and or cysteinecontaining glutathione (GSH) in combination with FTIR and mass spectrometry, they tried to extrapolate the chemical modifications on biological substrates. Using this method, several plasma sources such as the COSTJet, the kinPen, and the Cinogy DBD have already been compared $^{14,15,16}$.

4. To directly compare individual plasma sources, comparable control parameters must be established. Basic plasma parameters such as electron temperature, electron density, and the flux densities of reactive species are hard to measure in atmospheric pressure plasmas since such plasmas are often transient and their dimensions are small. Instead, external control parameters such as generator power, applied voltage or ignition, and arcing points are often used as a reference, especially when comparing results to simulations ${ }^{17,18}$. More recently, the measured electrical energy consumption has been used as a more reliable control parameter $19,20,21$.

Despite these efforts, comparing the results of different studies may still be impossible, simply due to the challenge of correctly applying a plasma source onto a surface. There are a vast number of prevalent pitfalls that have to be tackled when working with atmospheric pressure plasma applications such as the influence of external electric fields (compensation circuits), feedback loops between plasma and surrounding environment (shielded atmosphere), species transport (ionic wind) and control parameters (voltage, current, power).

The main objective of this work is to provide a thorough, detailed protocol on the application of the COST-Jet for surface treatments. The COST-Jet is a reliable plasma source that was developed for scientific reference purposes rather than for industrial or medical use. It provides reproducible discharge conditions and a broad database of available studies $^{22,23}$. The COST-Jet is based on a homogeneous, capacitively coupled RF-plasma. Because the electric field 
is confined perpendicular to the gas flow, charged species are mostly kept in the discharge region and do not interact with the target or the surrounding atmosphere. Additionally, the laminar gas flow ensures reproducible plasma chemical conditions in the plasma effluent.

In this paper, we will address the most common challenges and introduce possible solutions that have been used in the literature. These include proper gas supply, discharge control, ambient atmosphere influence, and surface preparation. Compliance with the protocol presented here should ensure the reproducibility and comparability of the measurements.

The protocol might also serve as an example for other atmospheric pressure sources. It must be refined for other jet plasma sources according to the individual gas flow and electric field configuration. Where applicable, we will try to point out possible adjustments to the protocol. The described steps should be considered and reported on when publishing studies applying atmospheric pressure plasmas to treated samples.

\section{Protocol}

\section{Feed gas supply and controlled atmosphere}

1. Set up the gas supply consisting of all-metal gas lines, avoiding any TPFE or similar plastics tubing ${ }^{24}$. Keep gas supply lines as short as possible to avoid any impurities and facilitate pumping of the gas supply system.

2. Choose the mass flow controllers used to provide the feed gas according to the typical gas flow rates of the COST-Jet. Use working gas with a purity of at least $99.999 \%$.

NOTE: The COST-Jet's primary working gas is helium. Operation can be realized at flow rates between 100 sccm and about $5000 \mathrm{sccm}$, with $1000 \mathrm{sccm}$ being the most common value.

3. Realize the admixture of reactive gases by a system consisting of multiple mass flow controllers. For smaller admixtures, use a counter-mixing unit to reduce the time needed for the mixing to complete ${ }^{25}$.

NOTE: Common admixtures are oxygen and nitrogen with a flow rate in the order of $5 \mathrm{sccm}(0.5 \%$ of the working gas).

4. Add a valve between the gas supply lines and the jet to prevent moist air entering the gas supply when the device is not in use as water is the most common and most problematic impurity in atmospheric pressure plasmas, critically influencing the plasma chemistry.

5. Clean the gas supply lines before the surface treatment, to reduce impurities in the tubing. To do so, either simply set a moderate gas flow of about $1000 \mathrm{sccm}$ helium and flush the supply lines or, preferably, repeatedly pump and refill the supply lines (about three times).

NOTE: When simply flushing the gas supply lines, multiple hours might be needed to clean the system, depending on the state of contamination.

6. Add a molecular sieve trap or cold trap (e.g., using liquid nitrogen) to the gas supply lines to further reduce the humidity in the feed gas.

7. If, instead, a controlled amount of water is desired as a reagent, add a bubbler to the system 26,27 .

8. Consider setting up a controlled atmosphere for your experiment as changes in the composition of the ambient atmosphere might influence chemical reactions in the plasma effluent.

NOTE: This effect is likely not very pronounced for the COST-Jet ${ }^{28}$, since the electric field configuration 
confines the plasma to the inside of the discharge channel but might play an important role for other CAP devices where the active plasma is partly outside the device.

\section{Assembly and setup of the device}

1. Connect the COST-Jet device to a gas supply. Directly connect the device to $1 / 4$ inch stainless steel Swagelok tubing. Use adapters for different tubing standards.

2. Connect the COST-Jet to the power supply using a shielded BNC cable equipped with an SMC connector.

3. Connect the integrated electrical probes to an oscilloscope to monitor voltage and current using a 50 Ohm resistor as termination.

4. Open the COST-Jet housing and connect a properly compensated commercial voltage probe to the powered copper line as well as a grounded part of the jet (e.g., the Swagelok gas tube) and the oscilloscope.

5. Perform a probe calibration routine: Apply a small voltage to the COST-Jet and tune the variable capacitor of the LC-circuit using a screwdriver to reach the optimum coupling (maximize measured voltage). Perform a voltage calibration by comparing the actual voltage (commercial probe) to the measured voltage (implemented probe) using linear regression and calculate a calibration constant. Remove the commercial voltage probe and close the COST-Jet housing.

6. Again, apply a small voltage to the COST-Jet and tune the variable capacitor of the LC-circuit using a screwdriver to reach the optimum coupling.

7. Ignite a plasma in the COST-Jet device: Firstly, set up a gas flow rate of approximately 1 slpm of helium using mass flow controllers (MFCs). Open the valve between the gas supply system and the COST-Jet last. Then, apply a low voltage to the electrodes and increase the amplitude until the plasma ignites.

8. If, upon the first ignition, the electrodes are unclean and impeding the ignition, apply a high initial voltage and quickly reduce it after ignition. Alternatively, use a spark gun to facilitate an easier first ignition.

9. Set the operation control parameters (gas flow, applied voltage) to the desired values.

10. Give the setup a little warm-up time to allow for thermal stabilization (approx. 20 minutes) to ensure stable and reproducible operation conditions.

11. To change the gas composition during the experiments, allow for an approximate 2 minute equilibration time depending on the gas supply setup.

NOTE: The COST-Jet is now ready for application.

\section{Power measurement}

1. Connect the oscilloscope monitoring the voltage and current applied to the COST-Jet to a computer.

2. Install the 'COST power monitor' software to the computer $^{29}$ which allows real-time power monitoring 11,19 .

3. Adjust the communication between the software and oscilloscope by implementing the required commands for controlling the specific oscilloscope.

4. Start the COST power monitor software and switch to the Settings panel. Fill in the correct channels connected to the oscilloscope and the calibration constant determined in step 2.4 . 
NOTE: The Find button can be used to automatically calculate the calibration factor if the commercial voltage probe is attached to the COST-Jet.

5. Change to the Sweep panel. Take a reference phase while the plasma is still off by pressing the Find button. Switch off the gas flow before this measurement and apply a voltage that is in the typical range of voltages used for the actual operation of the discharge as the plasma will not ignite in air due to much higher ignition voltage compared to noble gas dominated gas mixtures. Use this measurement to automatically correct for the relative phase shift between voltage and current probes, assuming a $90^{\circ}$ phase of the perfect capacitor here.

6. Press the Start and Pause button to start or pause the electrical measurements.

7. Operate the COST-Jet as desired. Use the actual electrical power calculated from voltage and current amplitudes as well as their phase shift, which are continuously displayed in the software for monitoring and as a control parameter.

\section{4. (Solid) surface treatment}

1. Set up a controlled atmosphere for your experiment. NOTE: In the case of the COST-Jet, the controlled atmosphere is less important than for sources with active plasma chemistry outside of the confined discharge channel.

2. Clean the gas supply lines as described in step 1.5.

3. Set the desired operating parameters and wait for approximately 20 minutes until the COST-jet reaches a stable temperature.

4. Choose the distance between the COST-Jet and the treated surface as the distance determines the amount of reactive species impinging on the treated surface ${ }^{30}$. Use an xyz-stage to mount the substrate for easy manipulation.

NOTE: For the COST-Jet, the safety gap adds one extra millimeter to the distance between the plasma discharge and the treated surface.

5. Start the treatment time: Either simply switch on the plasma or use a mechanical shutter. Be aware of a possible voltage overshoot during the switching event leading to a constricted discharge. For better control in the ms range, use a rotatable shutter.

6. Treat the sample for the desired amount of time and end the treatment time by switching off the plasma or by use of a shutter.

7. If necessary, check the gas flow pattern in front of the target using Schlieren imaging when treating a substrate as effects of surface charging, ion drag forces, or ambient air mixing due to buoyancy can influence the amount of reactive species reaching a surface.

\section{Liquid treatment}

1. Set up a controlled atmosphere for the experiment.

2. Clean the gas supply lines as described in step 1.5 .

3. Set the desired operating parameters and wait approximately 20 minutes for the COST-jet to reach a stable temperature.

4. Choose the distance between the COST-Jet and the treated liquid.

5. Pour the liquid to be treated into an adequate container. Use inert material to avoid reactions of potentially generated reactive species in the liquid with the 
container. Choose the size of the container according to the volume of liquid that is treated.

6. Consider the influence of the gas flow on the liquid surface: Depending on the gas flow rate, be aware of a concave meniscus that may form, thus changing the distance between plasma and liquid surface.

7. Start the treatment. Avoid pressure surges on the surface of the liquid caused by a sudden change in gas flow as this could cause liquid splashes into the discharge geometry, possibly causing a short circuit and certainly contaminating the plasma. Instead, use a mechanical shutter or slowly increase the gas flow.

8. Take into account mixing/stirring of the liquid due to friction between neutral gas flow and liquid surface as this influences transport processes and concentration profiles in the liquid. Additionally, depending on the treatment time, correct for the evaporation of liquid during the treatment (e.g., when calculating reaction constants). Depending on the plasma source, be aware of this evaporation possibly causing back coupling to the discharge, thus changing the plasma chemistry.

9. Please also consider that the reactivity with possible reagents in liquids is also affected by the surface activity of this agent. Thus, in some cases, surfactants might play an important role in the interaction between short-lived species and liquids.

\section{Representative Results}

Using the methods and equipment described above, we exemplary applied the COST-Jet to different surfaces and liquids. Figure 1 shows the experimental setup used for the treatment including the power supply, gas supply system, voltage and current probes as well as a controlled atmosphere and a mechanical shutter.

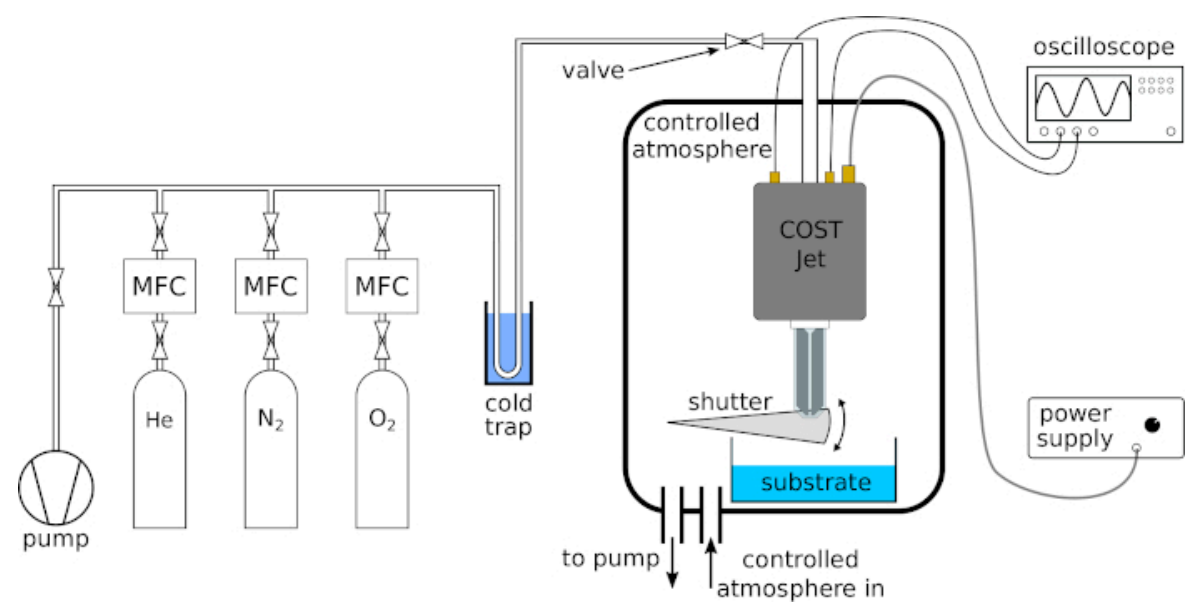

Figure 1: Experimental setup used for the plasma treatment of surfaces and liquids using the COST-Jet. A cold trap is used to purify the feed gas. The controlled atmosphere is realized by a pumped vacuum chamber at atmospheric pressure. The mechanical shutter facilitates the time management of solid and liquid surface treatment. The flexible stage allows controlling the distance between the plasma jet and the surface. Please click here to view a larger version of this figure. 
Using the voltage and current probe implemented in the COST-Jet, the dissipated electrical power can be calculated.

Figure 2 shows the measured electrical power in a helium plasma generated in five different COST-Jet devices using a gas flow of $1 \mathrm{slpm}$. All devices show similar behavior. The deviation between the different devices originates from the uncertainty of the power measurement as well as microscopic differences in the setups such as the electrode distance. More detailed measurements of reactive species (e.g., atomic oxygen and ozone), temperature and power as well as bactericidal activity measurements have been performed by Riedel $^{22}$.

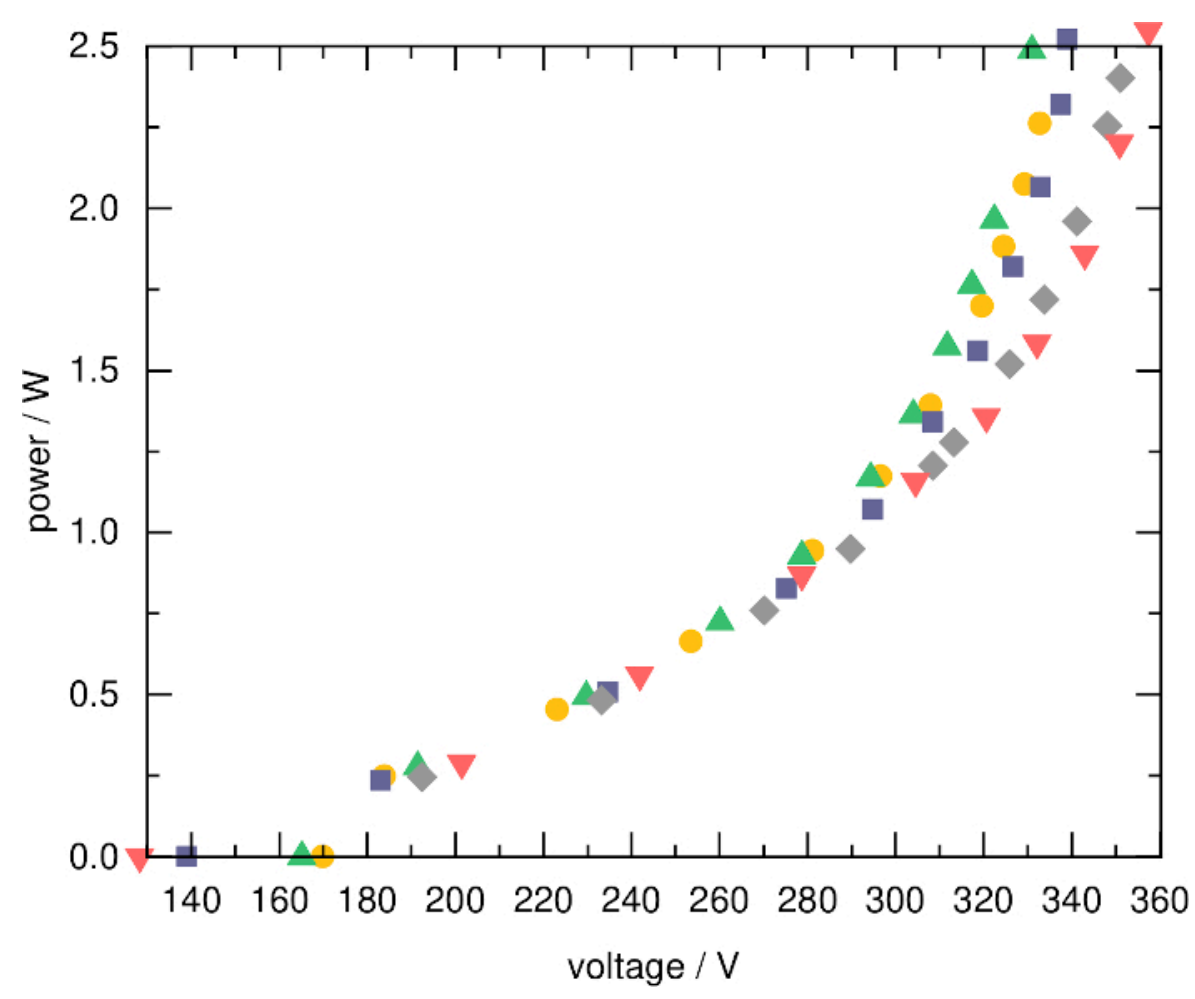

Figure 2: Dissipated power as a function of applied voltage in a helium plasma. The data represents five identical COST-Jet devices ${ }^{34}$. The small deviations at high voltages are due to uncertainties of the measurement as well as small deviations in the gas discharge channel geometry ${ }^{22}$. Please click here to view a larger version of this figure.

Figure 3 shows the etch profile of an a:C-H film for a 3 pattern shows a circular structure representing the cylindrical min treatment with the COST-Jet using a gas flow of 1.4 symmetry of the plasma effluent. Based on etch profiles slpm helium with an admixture of $0.5 \%$ oxygen measured in combination with numerical simulations, the surface loss using an imaging spectroscopic reflectometer ${ }^{31}$. The etch probability of atomic oxygen could be estimated. 

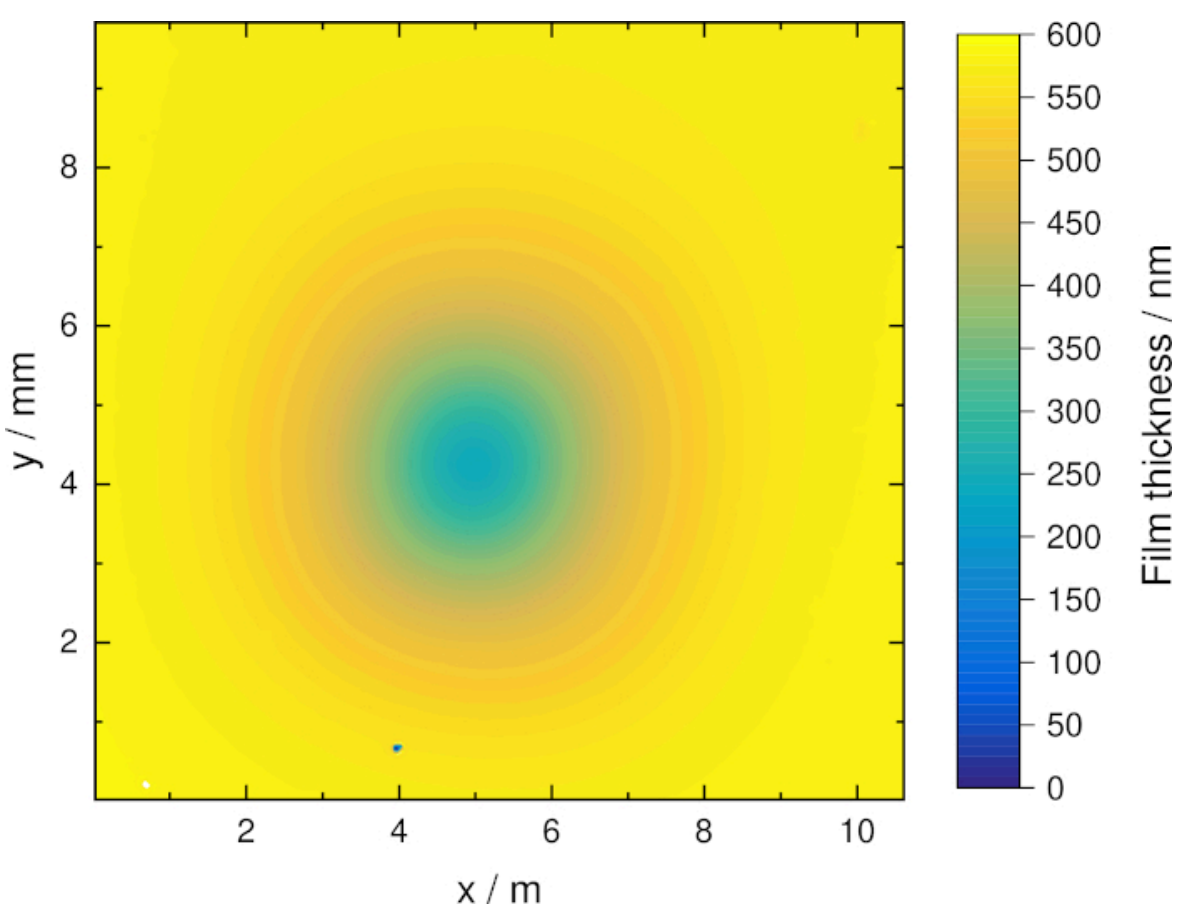

Figure 3: Etch profile of a plasma-treated a:C-H film. The dip in the film was etched using a gas mixture of $1.4 \mathrm{slm}$ helium with an admixture of $0.6 \%$ oxygen at a voltage of $230 \mathrm{~V}_{\mathrm{rms}}$ and a treatment time of 3 min. ${ }^{31}$ Please click here to view a larger version of this figure.

Figure 4 shows the occurring vortices in liquid caused by the gas stream impinging on the liquid surface. A laser sheet illuminating tracer particles in the liquid makes it possible to observe the trajectory and velocity of these particles via particle image velocimetry and therefore study the fluid flow $^{32}$. It is important to consider similar densities of the seeding particles and the fluid so that the trajectories of the particles represent the movement of fluid. With this visualization of the fluid flow measurements and numerical simulations can be compared ${ }^{33}$. The vortices are due to the surface friction between effluent gas flow and liquid surface. Figure 4 also shows the occurring depression of the liquid surface underneath the gas channel of the plasma jet, the socalled meniscus. It is visualized by a blue line. 


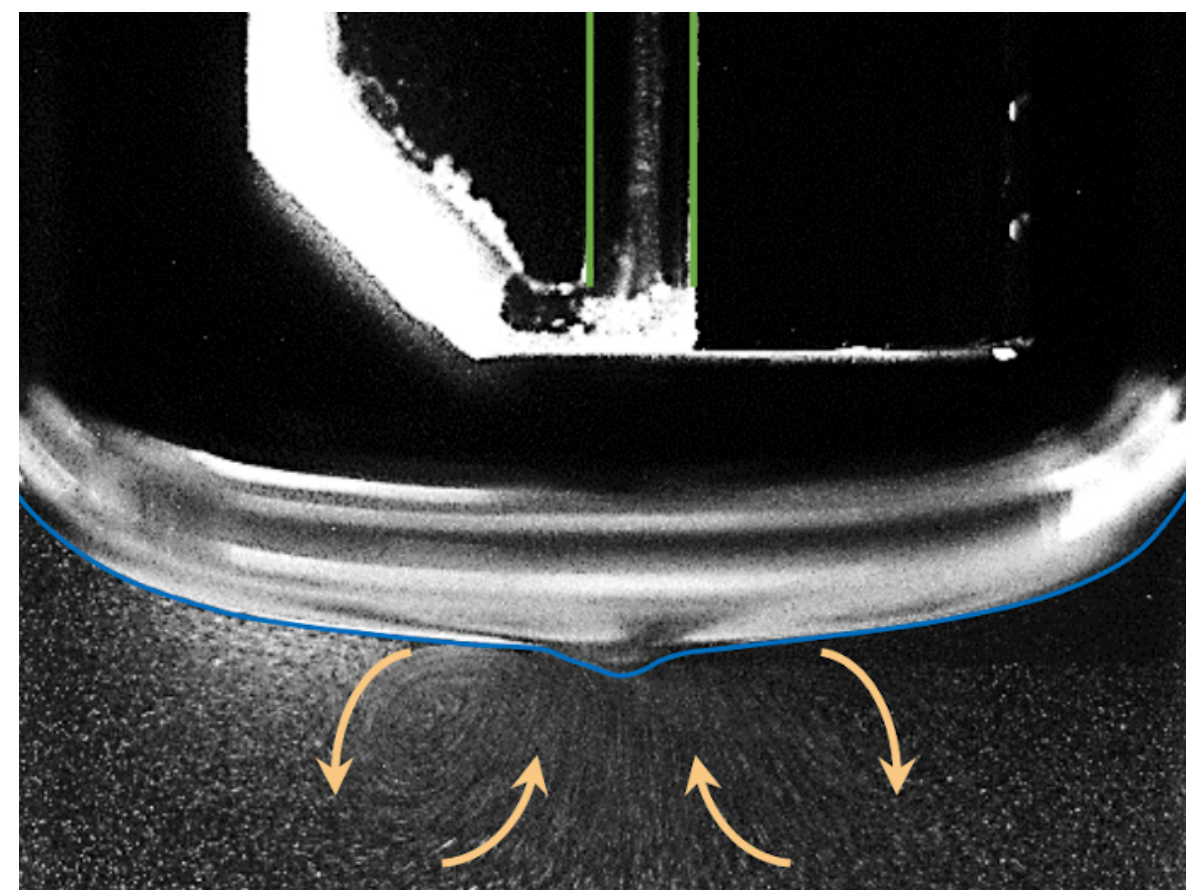

Figure 4: Photograph of illuminated cornstarch particles in $3 \mathrm{ml}$ of water stirred by the gas flow. The vortices are due to the surface friction between effluent gas flow and liquid surface. Please click here to view a larger version of this figure.

\section{Discussion}

Here, we demonstrate the use of an atmospheric pressure plasma jet for surface treatments of different materials. The experimental setup for an atmospheric pressure plasma jet can have a tremendous effect on the plasma parameters, chemistry, and performance and consequently influences the outcome of plasma treatments and is a critical step in the protocol.

As an example, the gas supply lines play an important role regarding the most common impurity in the feed gas of the plasma which is humidity. In particular, the production of reactive nitrogen species in the plasma is reduced while the reactive oxygen species production is favored, due to the low ionization energy of oxygen compared to water molecules and nitrogen ${ }^{35}$. Winter ${ }^{24}$ found out that feed gas humidity originating from water molecules on the surface of the inner tube is an order of magnitude higher using polymeric tubes compared to metal tubes due to the higher porosity and storage capacity. It can be reduced by flushing the lines with feed gas. However, drying the line by flushing takes a couple of hours. Therefore, polymeric tubing should be avoided or at least kept as short as possible. These findings are underlined by studies from Große-Kreul ${ }^{25}$. They compared the effect of polyamide and stainless steel tubing on the plasma chemistry using mass spectrometry. Their measurements confirm water cluster ion formation in the plasma due to water outgassing from polymeric tubes and faster drying times with metal tubes. Additionally, they investigated the effect of gas purification methods such as a molecular sieve trap and a liquid nitrogen 
cold trap on the plasma chemistry which helped to reduce the amount of impurities by about two orders of magnitude.

Instead of trying to purify the feed gas, there is also the approach of adding a controlled amount of humidity. As this intentional impurity then dominates over the natural impurities and thus controls the plasma chemistry, reproducible conditions are ensured as long as the amount of added humidity is precisely known.

For the ignition of the discharge, the applied voltage to the electrodes can usually simply be increased until the point of breakdown. However, depending on the surface conditions of the electrodes, sometimes a high voltage is necessary. To facilitate ignition, a high voltage spark gun can be used. This can also be useful when trying to ignite an argon discharge in the COST-Jet.

Before applying the COST-Jet to any surfaces, sufficient time should be allocated for the device to equilibrate. When set to the desired control parameters, the COST-Jet needs approximately 20 minutes to reach stable conditions ${ }^{11}$. During this time, the temperature of the device, the gas temperature as well as the plasma chemistry are reaching a steady state.

For comparison of scientific results, comparable plasma control parameters are necessary. For measuring the electrical input power, the COST power monitor can be used $^{29}$. The software is open-source and compatible with a range of different types of oscilloscopes. The software operates according to the principle described by Golda ${ }^{19}$.

In addition to the effect of feed gas humidity on the plasma chemistry, the transport of reactive species from the plasma to the substrate plays an important role in the effluent composition and is another critical step in the protocol. The surrounding atmosphere can influence the species created in the plasma on their way to the substrate. To minimize this influence, two different concepts are used: (i) Firstly, a controlled atmosphere can be set up that consists of the feed gas. Thus, the composition of the surrounding atmosphere can be kept constant. Depending on the purity level required for the treatment, the controlled atmosphere can be realized via protective housings equipped with a one-way valve to prevent overpressure. For higher purity levels, a vacuum chamber with a pump can be used. (ii) Secondly, a controlled atmosphere can be created by using a shielding gas curtain around the plasma effluent ${ }^{36,37}$. Usually, it consists of an inert gas, but it can also be varied according to the needs of the application.

Fortunately, for the COST-Jet, the influence of the surrounding atmosphere is comparably low. Using isotopic labeling, Gorbanev have shown that for a parallel-field configuration plasma jet, the reactive oxygen and nitrogen species reaching a liquid surface were formed in the plasma gas phase as well as in the region between the plasma nozzle and the sample 38,39 . In contrast, using the same technique for the COST-Jet, they found out that RONS almost exclusively originated from the plasma phase instead of the surrounding environment ${ }^{28}$. This is probably due to the electric field being confined to the plasma channel of the COST-Jet discharge. This makes the plasma discharge largely independent of its environment and gives it a certain remote character.

For a longitudinal electric field plasma jet, Darny et al. ${ }^{40}$ have shown that the polarity of the electric field modifies the gas flow pattern and thus also on the reactive species that reach a target due to ionic wind. The dependence of the reactive species density on the environment was confirmed 
by measurements by Stancampiano et al. ${ }^{7}$. They reported on the difference of the number of reactive species created in treated water depending on the electrical characteristics. To compensate for these differences, they had to create a compensating electrical circuit. This behavior is different for the COST-Jet: Figure 5 compares Schlieren images of the COST-Jet without an applied voltage and during operation for two different gas flow rates. The images were taken using a single mirror inline alignment as described by Kelly ${ }^{41}$. They show how the horizontally aligned COST-Jet effluent hits a flat glass substrate. Both images show the exact same gas flow pattern. This results from the lack of ionic wind due to the absence of charged species in the plasma effluent.
Additionally, the COST-Jet exhibits a very laminar flow pattern. Kelly ${ }^{41}$ showed Schlieren images similar to the ones presented in Figure 5, for various gas flow rates. Even at comparably high gas flow rates of 2 slpm, the plasma effluent shows no signs of turbulence. At very low gas flow rates of $0.25 \mathrm{slpm}$ and below, the buoyancy of the helium effluent begins to play a role. However, up to $4-5 \mathrm{~mm}$ distance from the nozzle, the ambient atmosphere does not influence the gas composition reaching the surface as demonstrated by Ellerweg using mass spectrometry ${ }^{17}$.

All of the above-mentioned characteristics add to the remote character of the COST-Jet. This makes it an ideal candidate for the controlled, comparable treatment of surfaces. 

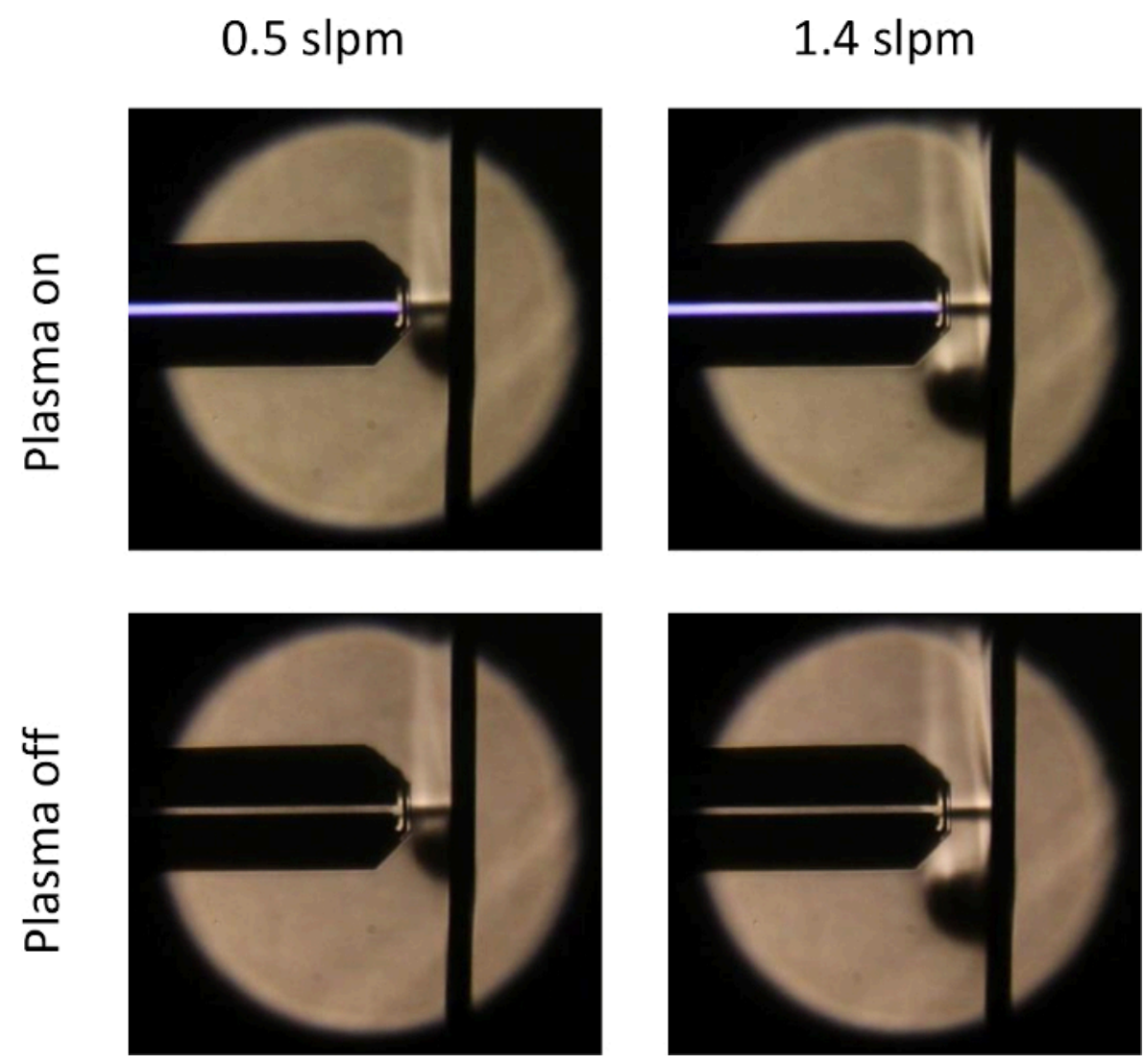

Figure 5: Schlieren images of the COST-Jet with and without applied voltage for two different gas flow rates. During plasma operation, the gas flow pattern exactly resembles the pattern with only the gas flow. Please click here to view a larger version of this figure.

Depending on the desired effect on the treated sample, the control parameters gas flow mixture, applied electrical power, and distance between plasma source and surface can be adjusted accordingly. For the COST-Jet, a broad literature database of studies investigating reactive species in the effluent exists. As an example, Willems ${ }^{30}$ measured the atomic oxygen density using mass spectrometry whereas Schneider $^{42}$ measured atomic nitrogen densities in the effluent.

The treatment of liquids with atmospheric pressure plasma can cause a variety of possible reaction mechanisms driven by reactive species, ions, photons, or electric fields. Due to the previously described characteristics of the COST-Jet, the effect of the electric field, ions, and photons are negligible compared to plasma sources where the plasma is in direct contact with liquids. Therefore, for studying the effect of short-lived reactive species like atomic oxygen on a phenol solution the COST-jet was used by Hefny ${ }^{43}$ and Benedikt ${ }^{44}$. Furthermore, the COST-Jet provides a convenient possibility to compare experiments and numerical simulations of liquid treatment ${ }^{28}$. As the interaction between plasma and liquid is 
dominated by the gas flow of reactive species from plasma to the liquid surface, the model complexity can be reduced.

The gas flow induced stirring of the liquid increases the reaction rate between plasma generated reactive species and the liquid. In contrast to surface treatments of solids, the convection of the liquid constantly changes the local concentration of reactants. Additionally, the reaction rates between plasma generated species with reactants in liquid are also affected by the surface activity of these reactants. With increasing surface activity, the concentration of the reactant at the liquid surface increases. These surfactants might play an important role in the reactivity of short-lived species generated by the plasma.

Next to stirring the gas flow impinging on the liquid surface also induces evaporation which must be considered. Using the COST-Jet with short treatment times the evaporation might play a minor role, although still has to be considered for calculating correct reaction rates. The discharge of the COST-Jet is not affected by the evaporation and therefore the plasma chemistry is also not affected. For different plasma sources, where e.g. the plasma is in direct contact with liquid, the plasma chemistry is changing significantly with evaporation as shown by Tian and Kushner ${ }^{45}$ for a dielectric barrier discharge. Also, for the kINPen, an effect of evaporations was determined ${ }^{46}$.

Besides these mentioned differences in plasma chemistry that need to be considered for different plasma sources, also the topology of the meniscus induced by gas stream on liquid surface changes. The depth of this meniscus is usually depending on the gas velocity. For plasma sources where the electrode configuration induces a significant electrical field reaching the liquid or even with a plasma in contact with the liquid, this meniscus can be elevated ${ }^{47,48}$. As shown, several effects need to be considered according to the used plasma source.

In the future, this protocol can be used to conduct and describe surface and liquid treatments using the COST-Jet. It is a stable, reproducible plasma source exhibiting a unique remote character amongst the plethora of different plasma jet designs. The same methods are not limited to the COST-Jet source only and can be modified and adapted to use with any cold atmospheric pressure plasma source.

\section{Disclosures}

The authors have nothing to disclose.

\section{Acknowledgments}

The authors thank Volker Rohwer (Institute of Experimental and Applied Physics, Kiel University) for help with the equipment. The work was supported by the DFG within CRC 1316 Transient Atmospheric Plasmas, in the project Cold atmospheric plasmas for the study of fundamental interaction mechanisms with biological substrates (projectID BE 4349/5-1), and in the project Plasma-generated nitric oxide in wound healing (project-ID SCHU 2353/9-1).

\section{References}

1. Morfill, G.E., Kong, M.G., Zimmermann, J.L. Focus on Plasma Medicine. New Journal of Physics. 11 (11), 115011 (2009).

2. Schlegel, J., Köritzer, J., Boxhammer, V. Plasma in cancer treatment. Clinical Plasma Medicine. 1 (2), 2-7 (2013).

3. Weltmann, K.D., Woedtke, T. von Plasma medicine —current state of research and medical application. 
Plasma Physics and Controlled Fusion. 59 (1), 14031 (2017).

4. Graves, D.B. Low temperature plasma biomedicine: A tutorial review. Physics of Plasmas. 21 (8), 80901 (2014).

5. Bruggeman, P.J. et al. Plasma-liquid interactions: A review and roadmap. Plasma Sources Science and Technology. 25 (5), 53002 (2016).

6. Simoncelli, E., Stancampiano, A., Boselli, M., Gherardi, M., Colombo, V. Experimental Investigation on the Influence of Target Physical Properties on an Impinging Plasma Jet. Plasma. 2 (3), 369-379 (2019).

7. Stancampiano, A. et al. Mimicking of human body electrical characteristic for easier translation of plasma biomedical studies to clinical applications. IEEE Transactions on Radiation and Plasma Medical Sciences. 1 (2019).

8. Nature Editorial. Reality check on reproducibility. Nature. 533 (7604), 437 (2016).

9. Baker, M. Is there a reproducibility crisis? Nature. 533, 452-454 (2016).

10. Begley, C.G., loannidis, J.P.A. Reproducibility in science: Improving the standard for basic and preclinical research. Circulation research. 116 (1), 116-126 (2015).

11. Golda, J. et al. Concepts and characteristics of the 'COST Reference Microplasma Jet'. Journal of Physics D: Applied Physics. 49 (8), 84003 (2016).

12. Mann, M.S., Schnabel, U., Weihe, T., Weltmann, K.D., Woedtke, T. von A Reference Technique to Compare the Antimicrobial Properties of Atmospheric Pressure Plasma Sources. Plasma Medicine. 5 (1), 27-47 (2015).

13. Kogelheide, F. et al. FTIR spectroscopy of cysteine as a ready-to-use method for the investigation of plasma- induced chemical modifications of macromolecules. Journal of Physics D: Applied Physics. 49 (8), 84004 (2016).

14. Lackmann, J.W. et al. Chemical fingerprints of cold physical plasmas - an experimental and computational study using cysteine as tracer compound. Scientific Reports. 8 (1), 7736 (2018).

15. Lackmann, J.W. et al. Nitrosylation vs. oxidation How to modulate cold physical plasmas for biological applications. PloS one. 14 (5), e0216606 (2019).

16. Ranieri, P. et al. GSH Modification as a Marker for Plasma Source and Biological Response Comparison to Plasma Treatment. Applied Sciences. 10 (6), 2025 (2020).

17. Ellerweg, D., Keudell, A. von, Benedikt, J. Unexpected O and $\mathrm{O}_{3}$ production in the effluent of $\mathrm{He} / \mathrm{O}_{2}$ microplasma jets emanating into ambient air. Plasma Sources Science and Technology. 21 (3), 34019 (2012).

18. Waskoenig, J. et al. Atomic oxygen formation in a radiofrequency driven micro-atmospheric pressure plasma jet. Plasma Sources Science and Technology. 19 (4), 45018 (2010).

19. Golda, J., Kogelheide, F., Awakowicz, P., Schulz-von der Gathen, V. Dissipated electrical power and electron density in an RF atmospheric pressure helium plasma jet. Plasma Sources Science and Technology. 28 (9), 95023 (2019).

20. Golda, J., Held, J., Gathen, V.S.v.d. Comparison of electron heating and energy loss mechanisms in an RF plasma jet operated in argon and helium. Plasma Sources Science and Technology. 29 (2), 25014 (2020). 
21. Beijer, P.A.C., Sobota, A., van Veldhuizen, E.M., Kroesen, G.M.W. Multiplying probe for accurate power measurements on an RF driven atmospheric pressure plasma jet applied to the COST reference microplasma jet. Journal of Physics D: Applied Physics. 49 (10), 104001 (2016).

22. Riedel, F. et al. Reproducibility of 'COST reference microplasma jets'. Plasma Sources Science and Technology. (2020).

23. COST Reference Microplasma Jet Homepage, www.cost-jet.eu. (2020).

24. Winter, J. et al. Feed gas humidity: a vital parameter affecting a cold atmospheric-pressure plasma jet and plasma-treated human skin cells. Journal of Physics D: Applied Physics. 46 (29), 295401 (2013).

25. Große-Kreul, S., Hübner, S., Schneider, S., Keudell, A. von, Benedikt, J. Methods of gas purification and effect on the ion composition in an RF atmospheric pressure plasma jet investigated by mass spectrometry. EPJ Techniques and Instrumentation. 3 (1), 6 (2016).

26. Benedikt, J. et al. Absolute $\mathrm{OH}$ and $\mathrm{O}$ radical densities in effluent of a He/H\$_ $\$$ O micro-scaled atmospheric pressure plasma jet. Plasma Sources Science and Technology. 25 (4), 45013 (2016).

27. Willems, G., Benedikt, J., Keudell, A. von Absolutely calibrated mass spectrometry measurement of reactive and stable plasma chemistry products in the effluent of a $\mathrm{He} / \mathrm{H} 2 \mathrm{O}$ atmospheric plasma. Journal of Physics D: Applied Physics. 50 (33), 335204 (2017).

28. Gorbanev, Y. et al. Combining experimental and modelling approaches to study the sources of reactive species induced in water by the COST RF plasma jet.
Physical chemistry chemical physics: PCCP. 20 (4), 2797-2808 (2018).

29. Held, J. mimurrayy/COST-power-monitor v0.9.2 (Version v0.9.2): Zenodo. (2019).

30. Willems, G. et al. Corrigendum: Characterization of the effluent of a $\mathrm{He} / \mathrm{O} 2$ micro-scaled atmospheric pressure plasma jet by quantitative molecular beam mass spectrometry (2010 New J. Phys.12 013021). New Journal of Physics. 21 (5), 59501 (2019).

31. Mokhtar Hefny, M., Nečas, D., Zajíčková, L., Benedikt, J. The transport and surface reactivity of $\mathrm{O}$ atoms during the atmospheric plasma etching of hydrogenated amorphous carbon films. Plasma Sources Science and Technology. 28 (3), 35010 (2019).

32. Grant, I. Particle image velocimetry: A review. Proceedings of the Institution of Mechanical Engineers, Part C: Journal of Mechanical Engineering Science. 211 (1), 55-76 (2016).

33. Semenov, I.L., Weltmann, K.D., Loffhagen, D. Modelling of the transport phenomena for an atmospheric-pressure plasma jet in contact with liquid. Journal of Physics D: Applied Physics. 52 (31), 315203 (2019).

34. Golda, J. Cross-correlating discharge physics, excitation mechanisms and plasma chemistry to describe the stability of an RF-excited atmospheric pressure argon plasma jet. Dissertation. Ruhr-Universität Bochum. Bochum (2017).

35. Lietz, A.M., Kushner, M.J. Molecular admixtures and impurities in atmospheric pressure plasma jets. Journal of Applied Physics. 124 (15), 153303 (2018).

36. Reuter, S. et al. Controlling the Ambient Air Affected Reactive Species Composition in the Effluent of an Argon 
Plasma Jet. IEEE Transactions on Plasma Science. 40 (11), 2788-2794 (2012).

37. Reuter, S. et al. From RONS to ROS: Tailoring Plasma Jet Treatment of Skin Cells. IEEE Transactions on Plasma Science. 40 (11), 2986-2993 (2012).

38. Gorbanev, Y., O'Connell, D., Chechik, V. Non-Thermal Plasma in Contact with Water: The Origin of Species. Chemistry (Weinheim an der Bergstrasse, Germany). 22 (10), 3496-3505 (2016).

39. Gorbanev, Y., Soriano, R., O'Connell, D., Chechik, V. An Atmospheric Pressure Plasma Setup to Investigate the Reactive Species Formation. Journal of visualized experiments. (117), 54765 (2016).

40. Darny, T. et al. Plasma action on helium flow in cold atmospheric pressure plasma jet experiments. Plasma Sources Science and Technology. 26 (10), 105001 (2017).

41. Kelly, S., Golda, J., Turner, M.M., Schulz-von der Gathen, V. Gas and heat dynamics of a micro-scaled atmospheric pressure plasma reference jet. Journal of Physics D: Applied Physics. 48 (44), 444002 (2015).

42. Schneider, S., Dünnbier, M., Hübner, S., Reuter, S., Benedikt, J. Atomic nitrogen: A parameter study of a micro-scale atmospheric pressure plasma jet by means of molecular beam mass spectrometry. Journal of Physics D: Applied Physics. 47 (50), 505203 (2014).

43. Hefny, M.M., Pattyn, C., Lukes, P., Benedikt, J. Atmospheric plasma generates oxygen atoms as oxidizing species in aqueous solutions. Journal of Physics D: Applied Physics. 49 (40), 404002 (2016).

44. Benedikt, J. et al. The fate of plasma-generated oxygen atoms in aqueous solutions: Non-equilibrium atmospheric pressure plasmas as an efficient source of atomic O(aq). Physical Chemistry Chemical Physics. 20 (17), 12037-12042 (2018).

45. Tian, W., Kushner, M.J. Atmospheric pressure dielectric barrier discharges interacting with liquid covered tissue. Journal of Physics D: Applied Physics. 47 (16), 165201 (2014).

46. Hansen, L. et al. Influence of a liquid surface on the NO $x$ production of a cold atmospheric pressure plasma jet. Journal of Physics D: Applied Physics. 51 (47), 474002 (2018).

47. van Rens, J.F.M. et al. Induced Liquid Phase Flow by RF Ar Cold Atmospheric Pressure Plasma Jet. IEEE Transactions on Plasma Science. 42 (10), 2622-2623 (2014).

48. Bruggeman, P., Graham, L., Degroote, J., Vierendeels, J., Leys, C. Water surface deformation in strong electrical fields and its influence on electrical breakdown in a metal pin-water electrode system. Journal of Physics $D$ : Applied Physics. 40 (16), 4779-4786 (2007). 